\title{
Universiteit
}

Leiden

The Netherlands

\section{Hydrogen a relevant shallow donor in Zinc Oxide}

Hoffmann, D.M.; Hofstaetter, A.; Leiter, F.; Zhou, H.; Henecker, F.; Meyer, B.K.; ... ; Baranov, P.

\section{Citation}

Hoffmann, D. M., Hofstaetter, A., Leiter, F., Zhou, H., Henecker, F., Meyer, B. K., ... Baranov, P. (2002). Hydrogen a relevant shallow donor in Zinc Oxide. Physical Review Letters, 88(4), 45504. doi:10.1103/PhysRevLett.88.045504

Version: $\quad$ Not Applicable (or Unknown)

License: $\quad$ Leiden University Non-exclusive license

Downloaded from: https://hdl.handle.net/1887/65481

Note: To cite this publication please use the final published version (if applicable). 


\title{
Hydrogen: A Relevant Shallow Donor in Zinc Oxide
}

\author{
Detlev M. Hofmann, Albrecht Hofstaetter, Frank Leiter, Huijuan Zhou, Frank Henecker, and Bruno K. Meyer \\ I. Physikalisches Institut, Heinrich-Buff-Ring 16, Justus-Liebig-Universität Giessen, D-35392 Giessen, Germany
}

Sergei B. Orlinskii and Jan Schmidt

Huygens Laboratory, Leiden University, P.O. Box 9504, 2300 RA Leiden, The Netherlands

Pavel G. Baranov

A. F. Ioffe Physico-Technical Institute, RAS, 194021 St. Petersburg, Russia

(Received 20 June 2001; published 10 January 2002)

\begin{abstract}
Electron paramagnetic resonance and Hall measurements show consistently the presence of two donors $(D 1$ and $D 2)$ in state-of-the-art, nominally undoped $\mathrm{ZnO}$ single crystals. Using electron nuclear double resonance it is found that $D 1$ shows hyperfine interaction with more than 50 shells of surrounding ${ }^{67} \mathrm{Zn}$ nuclei, proving that it is a shallow, effective-mass-like donor. In addition $D 1$ exhibits a single interaction with a $\mathrm{H}$ nucleus $\left(a_{H}=1.4 \mathrm{MHz}\right)$, thus $\mathrm{H}$ is the defining element. It is in agreement with the prediction of Van de Walle [Phys. Rev. Lett. 85, 1012 (2000)] that $\mathrm{H}$ acts as a donor in ZnO. The concentration of $D 1$ is $6 \times 10^{16} \mathrm{~cm}^{-3}$ emphasizing its relevance for carrier statistics and applications.
\end{abstract}

PACS numbers: 61.72.Vv, 71.55.Gs, 76.70.Dx

Despite the long history of research on $\mathrm{ZnO}$ the question of the $n$-type conductivity of nominally undoped crystals is still debated. In the discussion of the nature of the responsible shallow donors the complete spectrum of possibilities is under consideration, from residual impurities such as halogen atoms or group-III elements to intrinsic defects (Zn interstitials or the oxygen vacancy) [1]. In addition, and most recently, a new and unexpected possibility has been proposed from first-principles investigations, namely, that hydrogen is the cause of the doping [2]. This would be substantially different from the behavior of hydrogen in other semiconductors where it counteracts the prevailing type of conductivity and where it is widely used as a passivating species. Thus if experiments could support this prediction, and show that $\mathrm{H}$ causes a shallow donor in $\mathrm{ZnO}$ in relevant concentrations it would have serious impact on the growth methods as well as the technologies for devices which demand a specific conductivity type and carrier concentration. This is of special importance in view of the recently achieved $p$-type conductivity of $\mathrm{ZnO}$ which brought the material into the discussion as a serious alternative to the GaN-based blue/green optoelectronic emitters [3].

One of the most powerful methods to identify the chemical nature as well as the concentration of impurities and native defects in solid state materials is electron paramagnetic resonance (EPR) and electron nuclear double resonance (ENDOR) spectroscopy. We have used continuous wave $9 \mathrm{GHz}$ and, to enhance spectral resolution, pulsed $95 \mathrm{GHz}$ EPR and ENDOR spectroscopy [4]. We find that two donors are present in state-of-the-art nominally undoped $\mathrm{ZnO}$ single crystals which give rise to a single EPR line at $9 \mathrm{GHz}$, but two EPR lines at $95 \mathrm{GHz}$. One of these donors shows, in addition to the expected interaction with surrounding ${ }^{67} \mathrm{Zn}$ nuclei, a well-resolved interaction with a single ${ }^{1} \mathrm{H}$ nucleus. Thus our experimental results support the theoretical prediction that hydrogen can be a cause of the $n$-type conductivity of $\mathrm{ZnO}$.

The results of our temperature-dependent Hall measurements are very similar to those of Look et al. [5] who investigated $\mathrm{ZnO}$ crystals originating from the same source (Eagle-Picher). From the analysis of the Hall data we find two donor activation energies of $E_{D 1}=$ $(35 \pm 5) \mathrm{meV}$ and $E_{D 2}=(66 \pm 5) \mathrm{meV}$ and concentrations of $(6 \pm 2) \times 10^{16} \mathrm{~cm}^{-3}$ for $D 1$ and $(4 \pm 2) \times$ $10^{16} \mathrm{~cm}^{-3}$ for $D 2$.

In the $9 \mathrm{GHz}$ EPR experiments the shallow donors show up as a single line, see inset of Fig. 1. Its halfwidth is $0.2 \mathrm{mT}$, with $g$-values of $g_{\|}=1.957$ and $g_{\perp}=1.956$

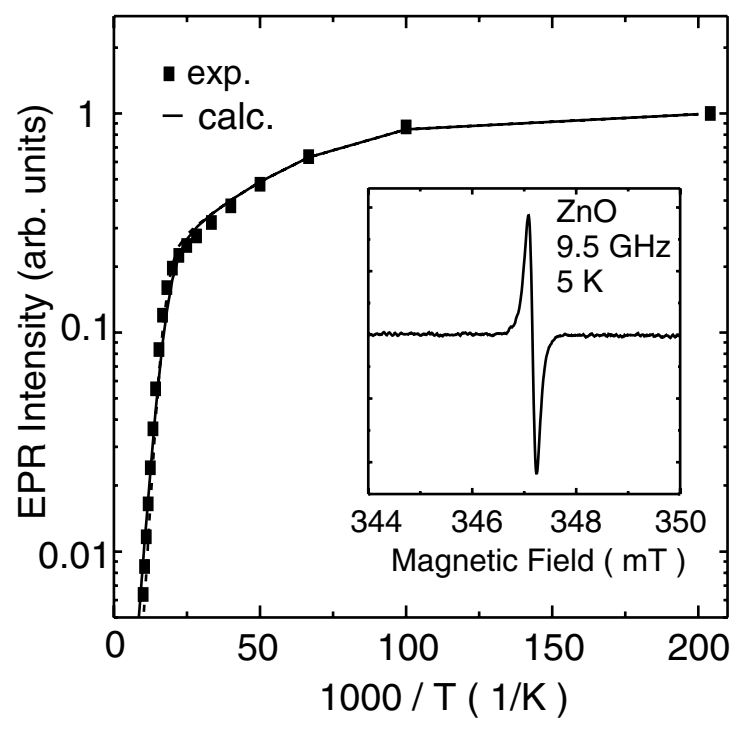

FIG. 1. The $9.5 \mathrm{GHz}$ EPR signal of the shallow donors in $\mathrm{ZnO}$ (inset) and the dependence of its intensity on temperature. The solid line shows the result of the calculation according to Eq. (1). 
(with respect to the crystal $c$ axis), in agreement with published data on shallow donors in $\mathrm{ZnO}$ [6-8]. To investigate whether this EPR signal corresponds to two donors as suggested by the Hall data we have carried out two types of experiments.

First, we determined the concentration of donors by comparison to a reference standard. It turned out that the $9 \mathrm{GHz}$ EPR signal corresponds to $(1.0 \pm 0.2) \times$ $10^{17} \mathrm{~cm}^{-3}$ donors, in good agreement with the total concentration of the two donors. Second, we investigated the temperature dependence of the EPR signal intensity (see Fig. 1). At low temperatures both donors should be in the paramagnetic $D^{0}$ state. When the temperature is increased the donor electrons are released to the conduction band leaving them in the positive diamagnetic charge state $D^{+}$, thus causing a decrease of the EPR signal. To analyze this behavior we used the following expression:

$$
I / I_{0}=\left(1+\sum_{i} K_{i} e^{-E_{i} / 2 k_{B} T}\right)^{-1},
$$

with $E_{i}$ the thermal activation energy of process $i$ with the prefactor $K_{i}$. For temperatures below $40 \mathrm{~K}$ the EPR intensity varies according to a rather small activation energy of $E_{1}=(4 \pm 2) \mathrm{meV}\left(K_{1}=6 \pm 3\right)$ which is likely to be related to weak potential fluctuations in the samples. For temperatures above $40 \mathrm{~K}$ we can fit the decrease of the EPR signal in two ways, either by one thermally activated process with $E_{2}=(47 \pm 4) \mathrm{meV}\left(K_{2}=\right.$ $\left.(5 \pm 2) \times 10^{4}\right)$, as previously obtained by Pöppel and Völkel [9] on ZnO powders, or by two activation energies as found in the Hall experiments, namely, $E_{2}=$ $(35 \pm 5) \mathrm{meV}$ and $E_{3}=(66 \pm 5) \mathrm{meV} \quad\left(K_{2}=K_{3}=\right.$ $4 \times 10^{3}$ ). Either model leads to a satisfying description of the experimental data. We favor the second case since it explains the Hall as well as the $9 \mathrm{GHz}$ EPR results.

Apparently the two donors have almost identical $g$ values which prohibit their separation in the $9 \mathrm{GHz}$ EPR experiments. However, with the pulsed $95 \mathrm{GHz}$ EPR spectrometer we are able to resolve two donor resonances (see Fig. 2). The corresponding sets of $g$-values for the two resonances are $g_{\|}=1.9569 \pm 0.00005$ and $g_{\perp}=$ $1.9552 \pm 0.00005$ for $D 1$, and $g_{\|}=1.9571 \pm 0.00005$ and $g_{\perp}=1.9555 \pm 0.00005$ for $D 2$ as determined from angular-dependent measurements. The assignment of the first set of $g$ values to the donor with an activation energy of $35 \mathrm{meV}$ and the second set to the deeper donor is based on the following arguments. With increasing binding energy the deviation of the $g$ values from that of the free electron value (2.0023) should become smaller, according to

$$
g-2.0023=\lambda / \Delta E,
$$

with $\lambda$ the spin-orbit coupling parameter and $\Delta E$ the energy separation to the next higher state. Further, one expects that with increasing binding energy the anisotropy of the $g$ value, i.e., $\Delta g=g_{\|}-g_{\perp}$, in the wurtzite crystal structure of $\mathrm{ZnO}$ becomes smaller. Also, the integrated

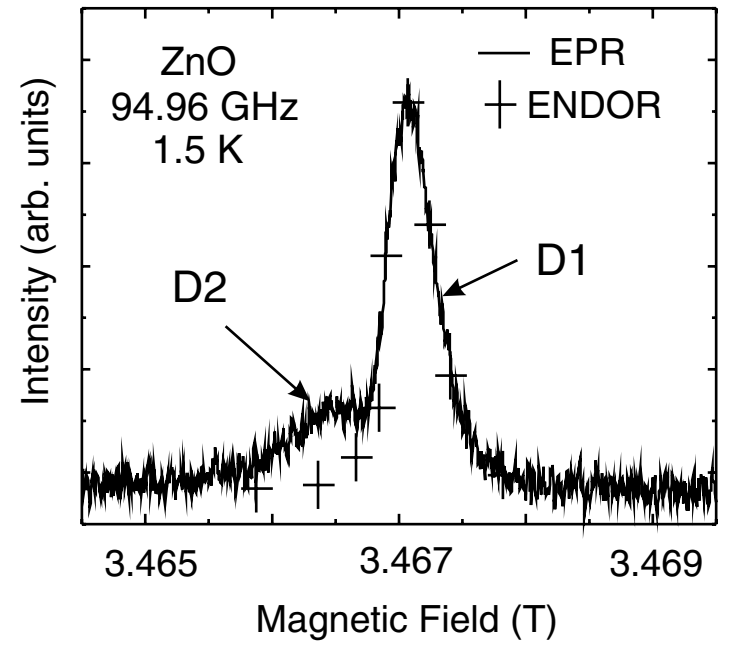

FIG. 2. EPR spectrum recorded with the magnetic field parallel to $c$. Owing to the higher resolution, the presence of two donor resonances $(D 1, D 2)$ is clearly visible. The symbols (crosses) give the intensity dependence of the ${ }^{1} \mathrm{H}$ ENDOR lines on the magnetic field.

EPR line intensity of $D 1$ is larger than that of $D 2$, in agreement with the concentration ratio of both donors as obtained from the Hall analysis.

Obviously, the EPR results do not provide information on the chemical nature of the donor species present since no resolved hyperfine (hf) structure is observed, as was the case for the In donors in $\mathrm{ZnO}$ [8]. We, therefore, performed ENDOR spectroscopy at $95 \mathrm{GHz}$ and at $1.5 \mathrm{~K}$ using a method which is based on the stimulated echo (SE) pulse sequence [10]. The spectrometer and its specific advantages for this work have been described in detail [4].

In Fig. 3 the high-frequency part of the ENDOR spectrum of the ${ }^{67} \mathrm{Zn}$ nuclei $(I=5 / 2$, abundance $4.11 \%)$ is presented with the magnetic field parallel to the crystal $c$ axis. The nuclear Zeeman frequency of ${ }^{67} \mathrm{Zn}$ is observable as a dip at 9.245 MHz. The ENDOR spectra proved to be isotropic apart from a few lines in the zinc spectrum which seem to exhibit an orientation dependence, and which are indicated by arrows.

For the understanding of the ENDOR results we will consider the isotropic hf interaction (Fermi contact term). The interaction constant $a_{i}$ reflects the spin density of the donor electron wave function $(\Psi)$ at the site of the nucleus $\left(r_{i}\right)$ and is given by

$$
a_{i}=\frac{8 \pi}{3} g_{e} \beta_{e} g_{n i} \beta_{n}\left|\Psi\left(r_{i}\right)\right|^{2},
$$

where $g_{e}$ is the electronic $g$ factor, $\beta_{e}$ is the electronic Bohr magneton, $g_{n i}$ is the $g_{n}$ factor of nucleus $i$, and $\beta_{n}$ is the nuclear magneton. The related ENDOR transitions have the following frequencies [11]:

$$
\nu_{\mathrm{ENDOR} i}=\frac{1}{h}\left|g_{n i} \beta_{n} B \pm \frac{a_{i}}{2}\right| .
$$

Therefore expression (4) predicts that each nucleus $i$ will give rise to two ENDOR transitions symmetrically placed 


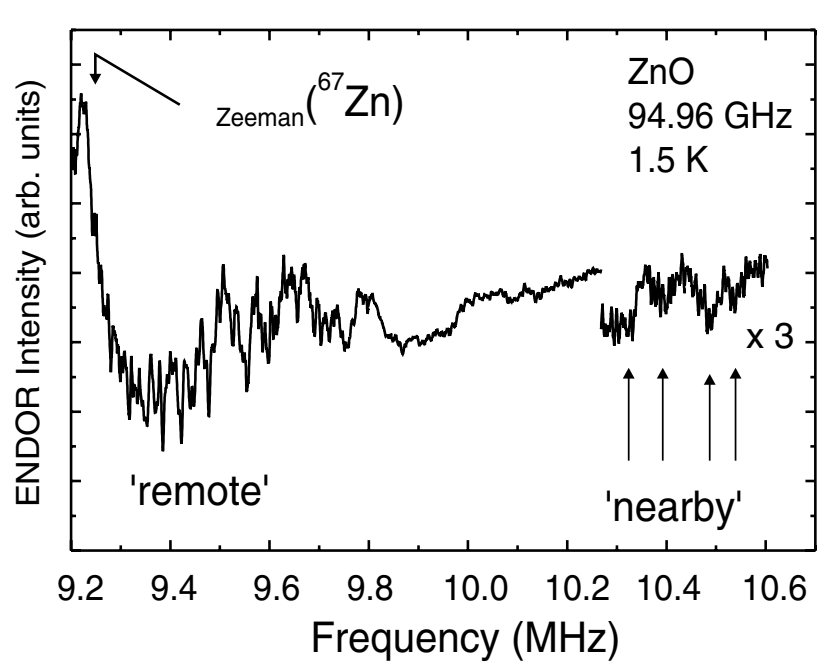

FIG. 3. The ENDOR spectrum of the ${ }^{67} \mathrm{Zn}$ nuclei at frequencies higher than the free Zeeman resonance at $9.245 \mathrm{MHz}$. The spectrum has been obtained using a $\pi / 2-\tau-\pi / 2-T-\pi / 2$ stimulated echo sequence with, in addition, an rf pulse during the interval $T$. The pulse lengths are $\pi / 2=0.15 \mu \mathrm{s}, \tau=0.4 \mu \mathrm{s}$, and $T=100 \mu \mathrm{s}$. The typical pulse length of the rf pulse is $100 \mu \mathrm{s}$. The signals close to the ${ }^{67} \mathrm{Zn}$ free Zeeman frequency are caused by the remote $\mathrm{Zn}$ nuclei. The four signals indicated by the arrows originate in nearby ${ }^{67} \mathrm{Zn}$ nuclei and exhibit an anisotropy.

around their nuclear Zeeman frequency $g_{n i} \beta_{n} B_{0} / h$ (when the quadrupole interaction is neglected). This behavior is indeed observed. The fact that a multitude of lines is present in Fig. 3 indicates that we are dealing with a delocalized electron which interacts with a large number of $\mathrm{Zn}$ nuclei. The same types of ENDOR spectra were observed for shallow electron centers in $\mathrm{AgCl}$ and $\mathrm{AgBr}$ crystals [12], where the hf interactions with $\mathrm{Ag}, \mathrm{Cl}$, and $\mathrm{Br}$ nuclei were observed.

For a quantitative analysis of the observed hf constants, expression (3) indicates that it is necessary to determine the spin density $\left|\Psi\left(r_{i}\right)\right|^{2}$ on each nucleus $i$. This problem has already been studied $[12,13]$. It was shown that, by orthogonalizing a suitable envelope function $\Phi$ to the cores of the lattice ions in order to allow for the Pauli principle, the spin density on nucleus $i$ may be written as an "amplification factor" $A_{i}$ times the density of the envelope function $\Phi$ on nucleus $i$. Thus $|\Psi(i)|^{2}=A_{i}|\Phi(i)|^{2}$. If the envelope function remains approximately constant within each ion core (which is the case for a strongly delocalized envelope function $\Phi$ ), the value of $A_{i}$ will depend only on the species of ion $i$, and not on its position in the lattice. We then rewrite Eq. (3) to

$$
a_{i}=\frac{8 \pi}{3} g_{e} \beta_{e} g_{n \mathrm{Zn}} \beta_{n} A_{\mathrm{Zn}}|\Phi(i)|^{2} .
$$

Here $A_{\mathrm{Zn}}$ is the "amplification factor" for $\mathrm{Zn}$.

To derive the spatial extension of the envelope function $\Phi(i)$ we assume that the ground state of the shallow donors in $\mathrm{ZnO}$ can be described by a hydrogenlike $1 s$ wave function $\Phi(r) \sim \exp \left(-r / r_{\mathrm{B}}\right)$ [14]. In the analysis of the
ENDOR results we will base our findings on a comparison with the ENDOR spectra of the shallow electron centers in $\mathrm{AgCl}$ [12] for which the amplification factor for $\mathrm{Ag}$ atoms was found to be 3200 . The reason for doing this is that both crystals have a mixed covalent and ionic bonding, the band gaps and the positions of the shallow donor energy levels in the crystals have about the same values, and the conduction bands are mainly created by $4 s$ electrons of $\mathrm{Zn}$ in $\mathrm{ZnO}$ and $5 s$ electrons of $\mathrm{Ag}$ in $\mathrm{AgCl}$. Further, there is information about the wave function density at the nucleus for $\mathrm{Ag}$ and $\mathrm{Zn}$ from Morton and Preston [15] and for $\mathrm{Ag}^{0}$ and $\mathrm{Zn}^{+}$ions in the host matrix $\mathrm{NaCl}$ [16]. Thus we can estimate the amplification factor for $\mathrm{Zn}$ to be about 1500 from the experimental value of 3200 for $\mathrm{Ag}$ in $\mathrm{AgCl}$ [12]. Here Clementi and Roetti [17] wave functions for $\mathrm{Ag}^{0}$ and $\mathrm{Zn}^{0}$ were used for the calculation of the overlap integral.

The extension $r_{D}$ of the shallow donor in $\mathrm{ZnO}$ can be obtained from effective mass theory (EMT) as $r_{D}=$ $\frac{\left(\varepsilon / \varepsilon_{0}\right)}{\left(m^{*} / m_{e}\right)} r_{B} \approx 1.5 \mathrm{~nm}$, with $\varepsilon=8.65$ the dielectric constant, $m^{*}=0.3 \times m_{e}$ the polaron mass in $\mathrm{ZnO}$, and $r_{B}=$ $0.053 \mathrm{~nm}$ the Bohr radius. The effective Bohr radius $\left(r_{D}\right)$ for the shallow donor in $\mathrm{ZnO}$ is almost equal to the radius of the shallow electron center in $\mathrm{AgCl}(1.7 \mathrm{~nm})$. Based on this similarity we can rationalize the distribution of the hf interactions which have been observed in the ENDOR spectrum of Fig. 3 where one can observe up to 50 shells of $\mathrm{Zn}$ atoms. It should be noted that the lattice constant for $\mathrm{ZnO}$ is about $20 \%$ smaller than in $\mathrm{AgCl}$, and that the ratio of the Bohr radii in $\mathrm{ZnO}$ and $\mathrm{AgCl}$ is about 0.8 . Thus the extension of the envelope function corrected for the lattice constant is almost the same. Within this volume the ${ }^{67} \mathrm{Zn}$ nuclei are positioned at random distances $r_{i}$, and they give rise to the large number of ENDOR resonances centered around the nuclear Zeeman frequency of ${ }^{67} \mathrm{Zn}\left(a_{i} \ll g_{n} \mu_{n} B_{0}\right)$. This analysis shows clearly that the donor electron wave function is effective-mass-like, i.e., we are dealing with shallow donors.

In order to obtain information on the chemical nature of the shallow donors, we performed ENDOR experiments on the common residual impurities ( $\mathrm{F}, \mathrm{Cl}, \mathrm{Br}$ and $\mathrm{Al}, \mathrm{Ga}, \mathrm{In}$ ). Despite the fact that the abundances of all isotopes of all these elements have nuclear spin, we were unable to detect any ENDOR transitions in these frequency ranges.

However, symmetrically placed around the nuclear Zeeman frequency of ${ }^{1} \mathrm{H}(147.6 \mathrm{MHz})$ we observe two ENDOR lines separated by $1.4 \mathrm{MHz}$ (Fig. 4). The two lines are isotropic and correspond to a hyperfine interaction constant $a_{H}=1.4 \mathrm{MHz}$ [see Eq. (3)]. Thus $\mathrm{H}$ is located in one well-defined position within the electron wave function of the shallow donor and not at random positions.

If we place the $\mathrm{H}$ nucleus at the (Coulomb) center of the shallow donor we can estimate the density of the envelope function $\Phi$. The hf constant of hydrogen in its $1 s$ state is $1420 \mathrm{MHz}$; an interaction constant of about $60 \mathrm{kHz}$ is predicted on the basis of the reduction of the density by the factor $\left(r_{B} / r_{D}\right)^{3}$. Since our ENDOR experiments give 


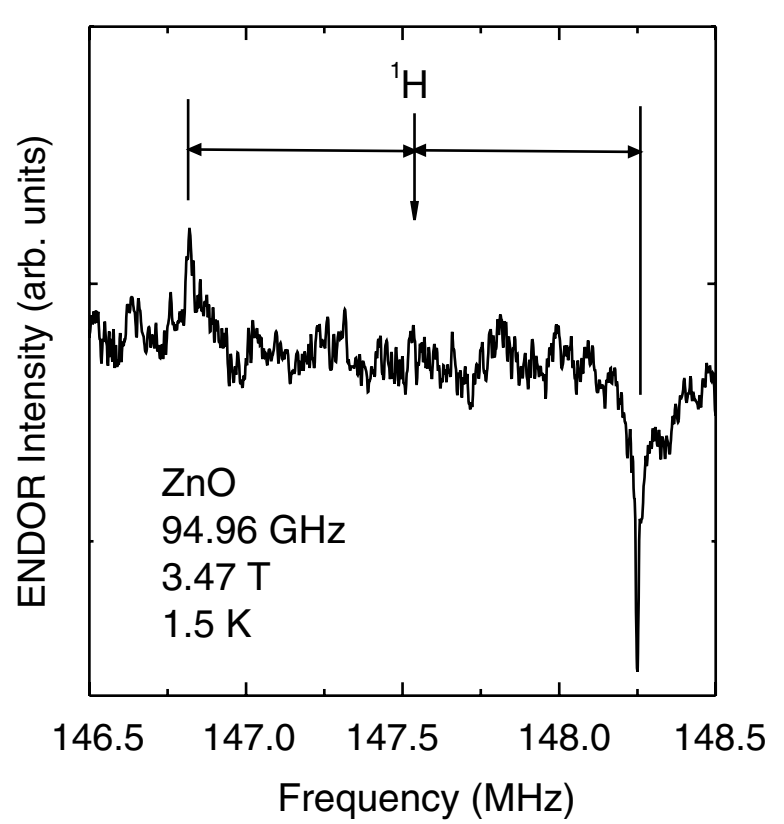

FIG. 4. The ENDOR spectrum of $\mathrm{H}$ of the $D 1$ donor in $\mathrm{ZnO}$. The conditions are the same as Fig. 3 (crystal $c$ axis parallel to the static magnetic field).

a value of $1.4 \mathrm{MHz}$, the amplification factor for $\mathrm{H}, A_{H}$, is about 20 which agrees well with the theoretical value of 15 derived in a way similar to that of $\mathrm{Zn}$. This seems to be a realistic value because the amplification factor for Li for $F$ centers in $\mathrm{LiF}$ was found to be 57 [13]. Thus it is very likely that the $\mathrm{H}$ nucleus is in the center of the shallow donor electron wave function, or at least in a position very close to the center. This is further supported by a comparison of our results with those of Block et al. [8] who observed the hf structure of the shallow In donor in $\mathrm{ZnO}$. The amplification factor for $\mathrm{In}$ in $\mathrm{ZnO}$ can be calculated to be 2500, in analogy to the amplification factors of $\mathrm{H}$ and $\mathrm{Zn}$ in $\mathrm{ZnO}$, and using the wave function density of $\mathrm{In}^{2+}$ in $\mathrm{KCl}$ [18]. Here the overlap integrals for $\operatorname{In}^{0}$ were used. To explain the experimental hf value of In we have to reduce the effective Bohr radius $r_{D}$ to $1.0-1.1 \mathrm{~nm}$. With this result we can estimate the ionization energy of the shallow In donor in $\mathrm{ZnO}$ to be approximately 2 times larger than that of the $\mathrm{H}$ donor in $\mathrm{ZnO}$. This is in agreement with the observation that the $g$ values of the In shallow donor are closer to that of the $D 2$ donor.

The ${ }^{1} \mathrm{H}$ ENDOR intensity is high at magnetic-field positions, where the $D 1$ EPR is the strongest (see Fig. 2), making it very likely that $\mathrm{H}$ is the cause of the $35 \mathrm{meV}$ donor in $\mathrm{ZnO}$. The origin of the $D 2$ donor so far remains unknown.

Figure 4 shows that the two ENDOR transitions have opposite signs. As shown in Ref. [19] it means that the sign of the hf interaction is positive and caused by direct spin density on the $\mathrm{H}$ nucleus. This finding is in line with the prediction of Van de Walle [2] that $\mathrm{H}^{+}$is present as the binding core for the shallow donor electron.
In summary, our EPR and ENDOR studies at $95 \mathrm{GHz}$ show that one of the two observed donor resonances is related to hydrogen. It is characterized by a thermal activation energy $E_{D 1}=(35 \pm 5) \mathrm{meV}$. The $\mathrm{H}$ nucleus is in the center or in a position close to the center of the shallow donor electron wave function. The observed hyperfine interactions with about 50 shells of $\mathrm{Zn}$ nuclei prove that it is a shallow, effective-mass-like donor with a Bohr radius of about $1.5 \mathrm{~nm}$. This donor is unintentionally present in commercially available $\mathrm{ZnO}$ in a concentration of about $(6 \pm 2) \times 10^{16} \mathrm{~cm}^{-3}$.

[1] D. C. Look, J. W. Hemsky, and J. R. Sizelove, Phys. Rev. Lett. 82, 2552 (1999), and references therein.

[2] C. G. Van de Walle, Phys. Rev. Lett. 85, 1012 (2000).

[3] M. Joseph, H. Tabata, and T. Kawai, Jpn. J. Appl. Phys. 38, L1205( 1999).

[4] A description of the $95 \mathrm{GHz}$ EPR/ENDOR setup can be found in J. A. J. Disselhorst, H. J. van der Meer, O. G. Poluektov, and J. Schmidt, J. Magn. Reson. A 115, 183 (1995).

[5] D. C. Look, D. C. Reynolds, J. R. Sizelove, R. L. Jones, C. W. Litton, G. Cantwell, and W. C. Harsch, Solid State Commun. 105, 399 (1998).

[6] J. Schneider and A. Räuber, Z. Naturforsch. Teil A 16, 712 (1961).

[7] M. Schulz, Phys. Status Solidi (a) K5, 27 (1975).

[8] D. Block, A. Herve, and R. T. Cox, Phys. Rev. B 25, 6049 (1982).

[9] A. Pöppel and G. Völkel, Phys. Status Solidi (a) 115, 247 (1989).

[10] W. B. Mims, in Electron Paramagnetic Resonance, edited by S. Geschwind (Plenum, New York, 1972).

[11] See, for example, J.-M. Spaeth, J. R. Niklas, and R. H. Bartram, Structural Analysis of Point Defects in Solids (Springer-Verlag, Berlin, 1992), p. 151.

[12] M. T. Bennebroek, A. Arnold, O. G. Poluektov, P. G. Baranov, and J. Schmidt, Phys. Rev. B 54, 11276 (1996), and references therein.

[13] B. S. Gourary and F. S. Adrian, Solid State Phys. 10, 127 (1960).

[14] K. W. Boer, Survey of Semiconductor Physics (Van Nostrand Reinhold, New York, 1990).

[15] J. R. Morton and K. F. Preston, J. Magn. Reson. 30, 577 (1978).

[16] P. G. Baranov, R. A. Zhitnikov, and N. I. Melnikov, Phys. Status Solidi 30, 851 (1968); P. G. Baranov, R. A. Zhitnikov, and V. A. Khramtsov, Phys. Status Solidi (b) 86, K67 (1978).

[17] E. Clementi and C. Roetti, At. Data Nucl. Data Tables 14, 177 (1974).

[18] P. G. Baranov and V.A. Khramtsov, Fiz. Tverd. Tela (Leningrad) 21, 1455 (1979) [Sov. Phys. Solid State 21, 839 (1979)].

[19] M. T. Bennebroek and J. Schmidt, J. Magn. Reson. 128, 199 (1997). 